\title{
The Visuo-Motor Pathway in the Local Circuit of the Rat Superior Colliculus
}

\author{
Tadashi Isa, Toshiaki Endo, and Yasuhiko Saito \\ Department of Integrative Physiology, National Institute for Physiological Sciences, Myodaiji, Okazaki 444-8585, Japan
}

Intrinsic circuit of the superior colliculus (SC), in particular the pathway from the optic tract (OT) to neurons in the intermediate layer (SGI), was investigated by whole-cell patch-clamp recording in slice preparations obtained from 17- to 24-d-old rats. Stimulation of the OT induced monosynaptic EPSPs in neurons in the superficial gray layer (SGS) and the optic layer (SO), and disynaptic or polysynaptic EPSPs in a majority of SGI neurons. Stimulation of the SGS induced monosynaptic or oligosynaptic EPSPs in the SGI neurons. Both the monosynaptic EPSPs induced in the SGS/SO neurons by stimulation of the OT and those induced in the SGI neurons by stimulation of the SGS were mediated by AMPA- and NMDA-type glutamate receptors. Thus, we have clarified the existence of the glutamatergic excitatory pathway from the OT to the SGI neurons via SGS and SO neurons. The EPSPs in the SGI neurons induced by stimulation of the OT or SGS were remarkably enhanced by bicuculline, suggesting that the signal transmission in this pathway is under strong suppression by the GABAergic system.

Key words: superior colliculus; local circuits; slice; patch clamp; intracellular staining; rat
The mammalian superior colliculus (SC) consists of several layers with distinct organization. The superficial layers [the stratum griseum superficiale (SGS) and the stratum opticum (SO)] receive visual input directly from the optic tract (OT) and indirectly from the primary visual cortex. There is a topographic representation of the contralateral visual field in the superficial layers (Cynader and Berman, 1972). The intermediate and deep layers [the stratum griseum intermediale (SGI) and the stratum griseum profundum (SGP)] receive nonvisual sensory inputs (Wallace and Stein, 1996) and indirect visual inputs from higher cortical visual areas (Harvey and Worthington, 1990; Harting et al., 1992). These layers send descending motor commands to the brainstem and spinal cord (Huerta and Harting, 1982). The deeper layers have a topographic representation of vector components of saccades (Robinson, 1972). This motor representation exists with similarly aligned multiple sensory representations. Because the spatial representations of the visual map in the superficial layers and motor map in the deeper layers coincide with each other, Robinson (1972) and Sprague (1975) proposed the existence of vertical connections from the superficial to deeper layers. However, this proposition was opposed by Edwards (1980), who on close anatomical inspection concluded that no significant connection exists between the two layers. Recent anatomical studies (Mooney et al., 1988; Rhoades et al., 1989; Behan and Appel, 1992; Hall and Lee, 1993; Lee and Hall, 1995) have again suggested the existence of the interlaminar connection. Mooney et al. (1992) suggested that superficial layers transmit

Received May 20, 1998; revised Aug. 3, 1998; accepted Aug. 5, 1998.

We express sincere thanks to Drs. Hiroshi Aizawa and Yasushi Kobayashi for comments on this manuscript, Professor S. Ozawa for valuable discussion, and Ms. Michi Seo, Junko Yamamoto, and Chika Kamada for excellent technical assistance. This study was supported by grants from the Ministry of Education, Science, Sports and Culture of Japan (Project No. 08458266, 08279207, and 09268238), Japan Science and Technology Corporation, Daiko Foundation, Naito Memorial Foundation, and Uehara Memorial Foundation.

Correspondence should be addressed to Dr. Tadashi Isa, Department of Integrative Physiology, National Institute for Physiological Sciences, Myodaiji, Okazaki 444-8585, Japan.

Copyright (C) 1998 Society for Neuroscience $\quad 0270-6474 / 98 / 188496-09 \$ 05.00 / 0$ visual inputs to deeper layers in electrophysiological experiments. Lee et al. (1997) suggested the existence of the interlaminar connection by observing EPSCs in SGI neurons after stimulation of the SGS in slices of the tree shrew. However, detailed information about the synaptic organization in the pathway is still lacking. In behavioral studies, it has been shown that reaction times of saccades reveal several distinct peaks in distribution. The shortest reaction time is on the order of $70 \mathrm{msec}$ in monkeys and $100 \mathrm{msec}$ in humans, and these saccades are called "express saccades" (Fischer and Boch, 1983; Fischer and Ramsperger, 1984). Fischer (1987) proposed that the presence of several peaks reflects the existence of several distinct pathways in the central process to generate saccade commands and that the shortest pathway is mediated by the SC. On the basis of this proposition we hypothesized that the signal is transmitted directly from the superficial to deeper layers of the SC in the case of express saccades but not in the case of regular saccades. If so, the signal transmission from superficial to deeper layers should be significantly modulated by the behavioral context. In the present study, we investigated the pathway from the OT to the SGI neurons in slices of the rat SC. It will be shown that the pathway exists and presumably is mediated by SGS or SO neurons or both. Furthermore, as a mechanism that regulates the transmission in the pathway, the functional significance of the GABAergic inhibition will be discussed.

\section{MATERIALS AND METHODS}

Slice preparations. Frontal slices of the SC ( $400 \mu \mathrm{m}$ thick) were prepared from young Wistar rats [postnatal day $(\mathrm{P})$ 17-24]. The animals were deeply anesthetized with ether and decapitated. The brains were quickly removed, submerged immediately in ice-cold sucrose Ringer's solution containing (in mM): 234 sucrose, $2.5 \mathrm{KCl}, 1.25 \mathrm{NaH}_{2} \mathrm{PO}_{4}, 10 \mathrm{MgSO}_{4}, 0.5$ $\mathrm{CaCl}_{2}, 26 \mathrm{NaHCO}_{3}$, and 11 glucose, and bubbled with $95 \% \mathrm{O}_{2}$ and $5 \%$ $\mathrm{CO}_{2}$ for 5-10 min. Slices were cut with a Microslicer (DTK-2000, Dosaka EM, Kyoto, Japan). They were then incubated in the standard Ringer's solution at room temperature for $>1 \mathrm{hr}$ before recording. The standard Ringer's solution contained (in $\mathrm{mM}$ ): $125 \mathrm{NaCl}, 2.5 \mathrm{KCl}, 2 \mathrm{CaCl}_{2}, 1$ $\mathrm{MgCl}_{2}, 26 \mathrm{NaHCO}_{3}, 1.25 \mathrm{NaH}_{2} \mathrm{PO}_{4}$, and 25 glucose, and was continuously bubbled with $95 \% \mathrm{O}_{2}$ and $5 \% \mathrm{CO}_{2}, \mathrm{pH}$ 7.4. After incubation, a 
A

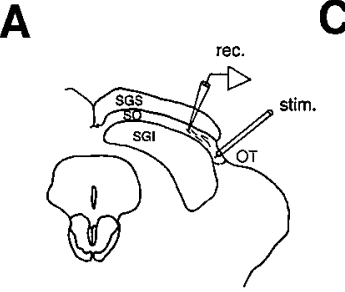

B

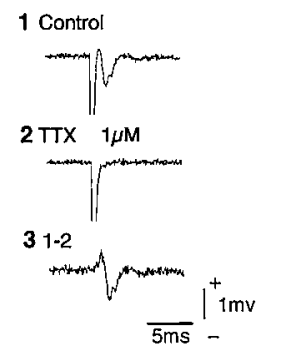

C

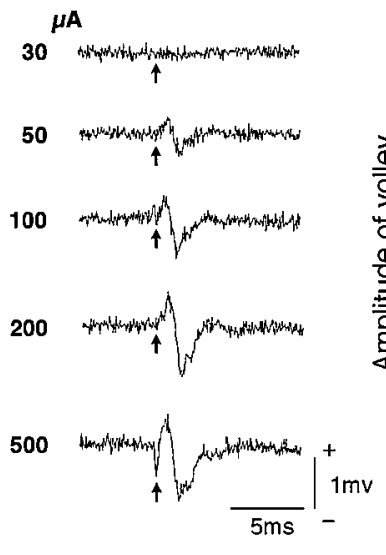

D

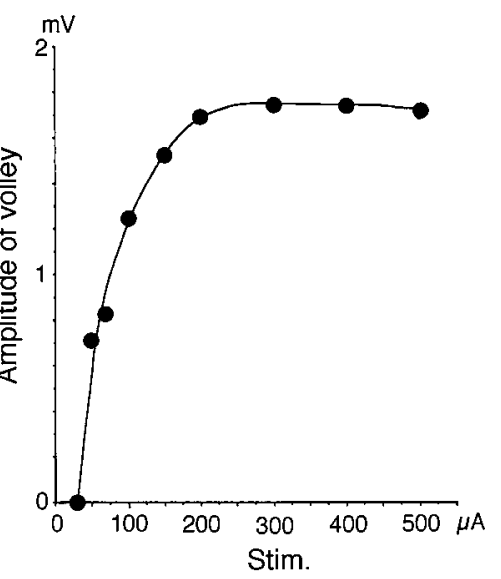

Figure 1. Conduction volley in the optic fibers. The OT was stimulated at the most lateral part of the SC, and the conduction volley was recorded in the SO medial to the stimulation site $(A)$. Stimulation of the OT induced a conduction volley (B1) that was completely abolished by application of $1 \mu \mathrm{M}$ tetrodotoxin $(T T X)(B 2)$. Subtraction of B2 from B1 eliminated the stimulus artifact and yielded a triphasic conduction volley (B3). The relationship between the stimulus strength and the amplitude of the conduction volley was investigated $(C)$, and plots of the amplitude of the volley against the stimulus strength revealed that the amplitude of the volley became saturated at $200 \mu \mathrm{A}$ stimulus in this example $(D)$. slice was mounted in a recording chamber on an upright microscope (Axioskop FS, Zeiss) and continuously superfused with the standard Ringer's solution at the rate of $3-5 \mathrm{ml} / \mathrm{min}$ by a peristaltic pump (Minipuls 3, Gilson, Villiers, France).

Whole-cell patch-clamp recording. Individual neurons in the SC were visualized with Nomarski optics with the use of a $40 \times$ water immersion objective. Whole-cell patch-clamp recordings (Hamill et al., 1981; Edwards et al., 1989) were obtained from neurons in the SGS, SO, and SGI under visual control of the patch pipettes using an EPC-7 patch-clamp amplifier (List, Darmstadt, Germany). The pipettes were filled with an internal solution containing (in $\mathrm{mm}$ ): 150 potassium gluconate, 6 (or 10) $\mathrm{KCl}, 0.2$ EGTA, $2 \mathrm{MgCl}_{2}, 2 \mathrm{Na}_{2} \mathrm{ATP}, 10 \mathrm{HEPES}$, and 0.1 spermine, $\mathrm{pH}$ 7.3. To stain the recorded neurons, biocytin $(5 \mathrm{mg} / \mathrm{ml})$ (Sigma, St. Louis, MO) was dissolved in the solution just before recording. Because the liquid junction potential between the standard Ringer's solution and the internal solution was estimated as $-10 \mathrm{mV}$, the actual membrane potential was corrected by this value. The osmolarity of the internal solution was 280-290 mOsm/1. The resistance of the electrodes was 2.5-7.0 M $\Omega$ in the bath solution. The series resistance during recording was $10-25$ $\mathrm{M} \Omega$. All recordings were performed at a bath temperature of $28-32^{\circ} \mathrm{C}$. Data were acquired using pClamp system (Axon Instruments, Foster City CA). Electrical stimulation of the optic tract was applied as a cathodal square-wave pulse with a duration of $200 \mu \mathrm{sec}$ using a concentric bipolar electrode (Clark Electromedical Instruments, Pangbourne, UK) or a resin-insulated tungsten needle monopolar electrode. The conduction volley of the optic fibers was recorded by a glass capillary containing $2 \mathrm{M} \mathrm{NaCl}$ (impedance: 0.5-2 $\mathrm{M} \Omega$ ). 6-Cyano-7nitroquinoxaline-2,3, dione (CNQX), D-2-amino-5-phosphonovalerate (APV) and bicuculline (Bic) were purchased from RBI (Natick, MA), and tetrodotoxin (TTX) was purchased from Sankyo (Tokyo, Japan). Records were filtered at a $3 \mathrm{kHz}$ bandwidth, and the sampling frequency was either 5 or $10 \mathrm{kHz}$.

Histological procedure. To visualize the recorded neurons by staining with biocytin (Horikawa and Armstrong, 1988), the patch pipettes were carefully detached from the cells after recording. The slices were fixed

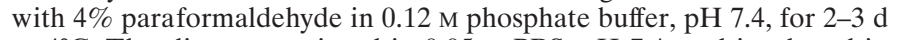
at $4^{\circ} \mathrm{C}$. The slices were rinsed in $0.05 \mathrm{M} \mathrm{PBS}, \mathrm{pH} \mathrm{7.4,} \mathrm{and} \mathrm{incubated} \mathrm{in}$ methanol containing $0.6 \% \mathrm{H}_{2} \mathrm{O}_{2}$ for $30 \mathrm{~min}$. After washes with PBS, the slices were incubated in avidin-biotin peroxidase complex solution (1\%) (Vector Laboratories, Burlingame, CA) containing 0.3\% Triton X-100 for $3 \mathrm{hr}$. After washes with PBS and $0.05 \mathrm{M}$ Tris-buffered saline (TBS), $\mathrm{pH} 7.6$, they were incubated in a TBS solution containing $0.01 \%$ diaminobenzidine tetrahydrochloride (DAB), $1 \%$ nickel ammonium sulfate, and $0.0003 \% \mathrm{H}_{2} \mathrm{O}_{2}$ for $30 \mathrm{~min}$. All procedures for visualization of biocytin were performed at room temperature. The slices were mounted on gelatin-coated slides, counterstained with cresyl violet, dehydrated, and then coverslipped. The morphological properties of stained cells were drawn using a camera lucida attached to a light microscope.

Determining appropriate stimulus strength of the optic tract. To investigate the input organization from the OT, the effects of the OT stimulation were investigated in neurons located in various layers of the SC. The OT was stimulated at the most lateral part of the SC, where the optic fibers compose a bundle (Fig. 1A). To exclude the possibility of current spread to the neural elements outside the OT, we determined the appropriate stimulus strength by measuring the stimulus current to induce the maximum conduction volley in the OT. As shown in Figure $1 A$, the conduction volley in the OT was measured at a point in the SO $300-500 \mu \mathrm{m}$ medial to the stimulation site. In the case of Figure $1 B 1$, the OT stimulation at $200 \mu \mathrm{A}$ evoked a large negative volley in the SO that disappeared after application of $1 \mu \mathrm{M}$ TTX, leaving the stimulus artifact (Fig. 1B2). The negative volley was resistant to CNQX (data not shown). Subtracting the record in Figure $1 B 2$ from that in Figure $1 B 1$ yielded a triphasic conduction volley by eliminating the stimulus artifact (Fig. 1B3). The peak-to-peak amplitudes of the conduction volley were measured at different stimulus strengths (Fig. 1C) and plotted (Fig. 1D). Figure $1 D$ shows that the conduction volley maximized at $200 \mu \mathrm{A}$ stimulation in this slice. This indicated that stimulation above this strength could activate the neural elements outside the OT and that the stimulus strength had to be set below $200 \mu \mathrm{A}$. The records in Figure 1 were obtained by using a concentric bipolar electrode. The usage of monopolar electrodes required current intensities to activate the maximal conduction volley in the OT similar to those required using the bipolar electrode. We performed this procedure in every slice, and all the results shown in this paper were obtained using the appropriate stimulus strength determined in this way.

\section{RESULTS}

\section{Input from the OT to neurons in the SGS}

The effect of the OT stimulation was investigated in 32 SGS neurons. Among them, 16 neurons were successfully stained with biocytin. The stained neurons consisted of five piriform cells, three narrow-field vertical cells, three stellate cells, three horizontal cells, and two wide-field vertical cells, according to the morphological characterization by Langer and Lund (1974).

Figure 2 shows the results recorded in a neuron in the SGS. This neuron projected dendrites in dorsoventral directions and appeared to be a narrow-field vertical cell (Langer and Lund, 1974). Extensive axonal projection was observed in the ventral part of the SGS and in the SO, and some extended farther to the SGI (Fig. 2A, thin lines). A large number of collaterals and bouton-like swellings were observed. This neuron showed repetitive spike firing with moderate spike frequency adaptation in response to depolarizing current pulses. In response to hyperpolarizing current pulses, this neuron showed inward rectification (Fig. 2B). Stimulation of the OT induced EPSPs with a fixed latency of $3.5 \mathrm{msec}$ (Fig. 2C1). When double shock stimuli were given with an interval of $4 \mathrm{msec}$, the latencies of the EPSP induced by the second stimulus (the onset is indicated by a 

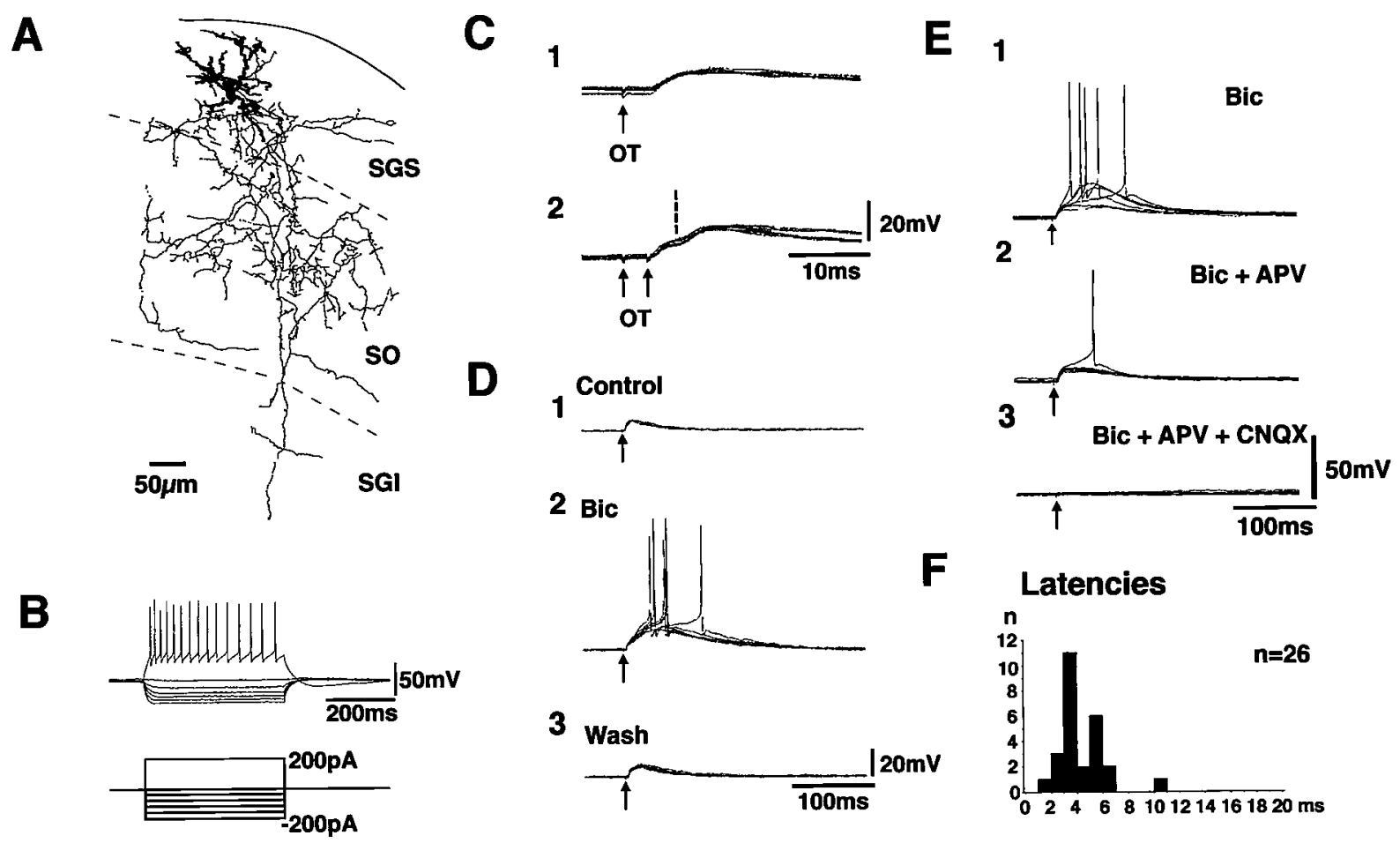

Figure 2. Synaptic potential induced by the OT stimulation in an SGS neuron. The morphology of the recorded neuron, stained with biocytin, is illustrated in $A$. Dendrites are drawn as thick lines. The axon and its collaterals are drawn as thin lines. $B$ shows the voltage responses of this neuron to various current pulses. The bottom traces indicate the injected currents. Synaptic potentials induced by single and double stimulus (at an interval of 4 $\mathrm{msec}$ ) of the OT are shown in $C 1$ and $C 2$, respectively. The effect of bath application of $10 \mu \mathrm{M}$ bicuculline (Bic) is shown in $D$. Effects of glutamate receptor antagonists, $10 \mu \mathrm{M} \mathrm{CNQX}$, and $50 \mu \mathrm{M}$ APV are shown in $E . F$ indicates distribution of latencies of the EPSPs induced by the OT stimulation in SGS neurons.

vertical broken line) were the same as those from the first (Fig. $2 C 2$ ). These results suggested strongly that the EPSPs were induced monosynaptically. The EPSPs could be induced in 26 neurons by the OT stimulation, and the latencies ranged from 1.9 to $10.6 \mathrm{msec}$ (Fig. $2 F$ ). Among these, monosynaptic EPSPs were observed in 21 neurons. The rise time of the monosynaptic EPSPs (the time between 10 and $90 \%$ peak) was measured for those with clear onset and single peak. The values ranged from 1.5 to 5.3 $\operatorname{msec}($ mean $\pm \mathrm{SE}=3.2 \pm 0.3 \mathrm{msec}, n=18$ ). Among the successfully stained neurons, monosynaptic EPSPs were observed in four of five piriform, three of three narrow-field vertical, two of three stellate, and two of three horizontal cells. Application of 10 $\mu \mathrm{M}$ Bic enhanced the late component of the EPSP with complete recovery after washing out (Fig. 2D). Application of $50 \mu \mathrm{M}$ APV reduced the amplitude of the late component, and additional application of $10 \mu \mathrm{M}$ CNQX completely abolished the EPSPs (Fig. 2E). All of these results indicate that various subgroups of SGS neurons receive monosynaptic excitatory input from the OT and that the excitatory transmission is mediated by both AMPAand NMDA-type glutamate receptors.

\section{Input from the OT to neurons in the SO}

The effects of the OT stimulation were investigated in 20 neurons in the SO. Figure 3 shows the records obtained in a wide-field vertical cell according to classification by Langer and Lund (1974) (Fig. 3A). This neuron extended dendrites divergently into the SGS and projected axons to the ventral part of the SO and farther ventrally into the SGI. This neuron showed regular firing in response to depolarizing current pulses and clear voltage sag in response to hyperpolarizing pulses and rebound depolarization after termination of the hyperpolarizing pulses (Fig. $3 B$ ). The voltage sag and the rebound depolarization in this type of neuron were shown to be caused by hyperpolarization-activated current (Saito and Isa, 1997). Among the 20 SO neurons recorded in the present study, 17 neurons were regarded as wide-field vertical cells according to the electrophysiological property (voltage sag). Ten of these neurons were successfully stained with biocytin, in which the classification was confirmed morphologically. In the neuron shown in Figure 3, stimulation of the OT induced EPSPs at a latency of $6.3 \mathrm{msec}$. The onsets of the EPSPs were constant over many sweeps. When double shock stimuli were applied with an interval of $4 \mathrm{msec}$, the EPSP component locked to the second stimulus (indicated with a vertical broken line) appeared to have the same latency as that induced by the first stimulus (Fig. 3C2). These results strongly suggested that the EPSPs were induced by monosynaptic linkage. The latency of the EPSPs ranged from 1.3 to $17.0 \mathrm{msec}$ in the 17 wide-field vertical cells (Fig. $3 F$, closed columns). Among these, monosynaptic EPSPs were observed in 16 neurons. In the remaining three neurons, which were not regarded as wide-field vertical cells (Fig. $3 F$, open columns), the OT stimulation induced EPSPs, which were not regarded as monosynaptic because of fluctuation of latencies. The rise time of the monosynaptic EPSPs (the time between 10 and 90\% peak) in the wide-field vertical cells was measured for those with distinct onset and single peak. The values ranged from 3.2 to $11.0 \mathrm{msec}$ (mean $\pm \mathrm{SE}=6.6 \pm 0.6 \mathrm{msec}, n=10$ ), much longer than those recorded in the SGS neurons. Application of Bic enhanced the late component of the EPSPs (Fig. 3D2,E2). The late component 


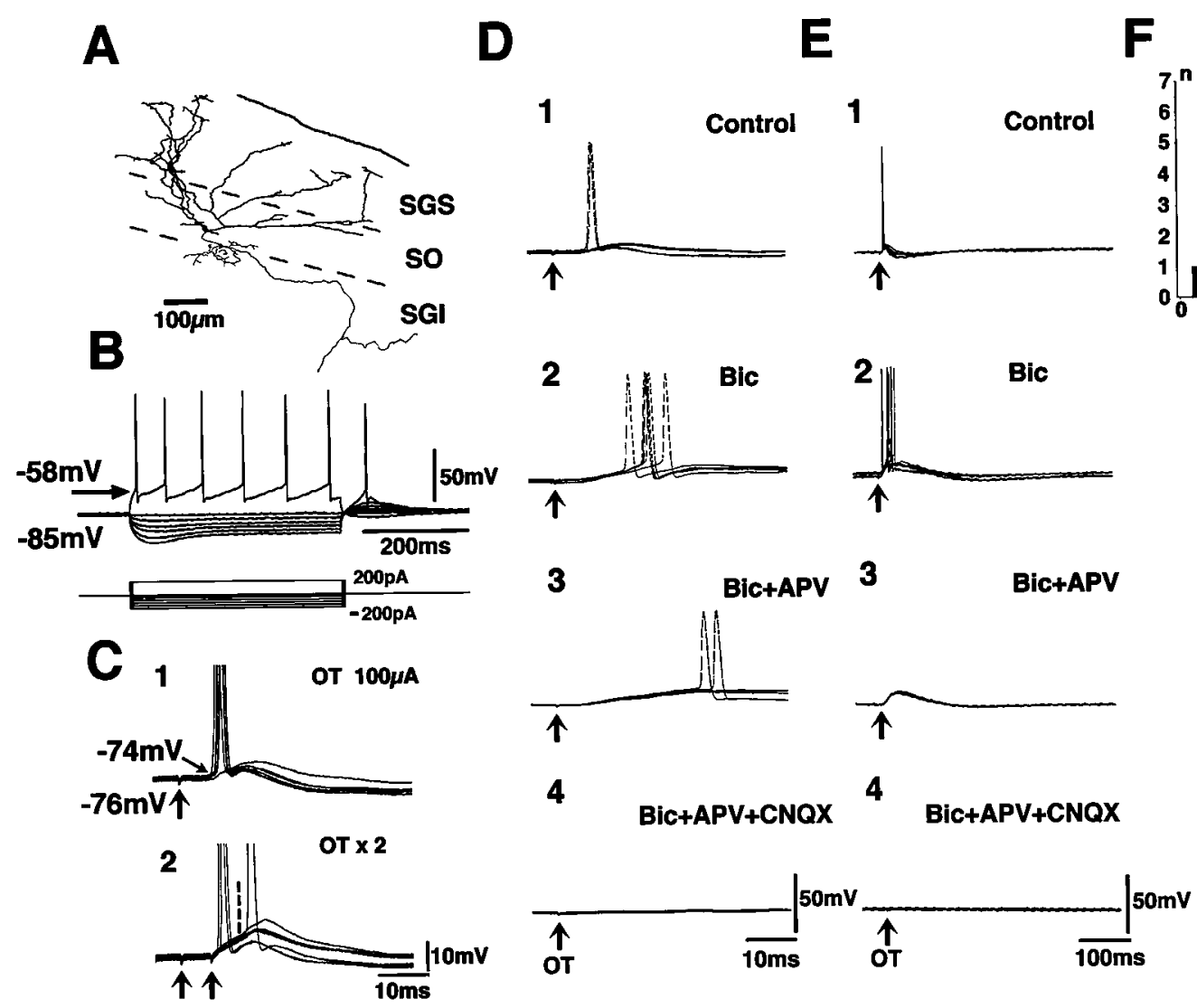

Figure 3. Synaptic potentials induced by the OT stimulation in an SO neuron. The morphology of the recorded neuron, stained with biocytin, is illustrated in $A$. Dendrites are drawn as thick lines. The axon and its collaterals are drawn as thin lines. $B$ shows the voltage responses of this neuron to various current pulses. Here, the threshold of the action potentials was approximately $-58 \mathrm{mV}$. Synaptic potential induced by the OT stimulation is shown in $C 1$. Action potentials were induced from the membrane potential of $-74 \mathrm{mV}$, overriding the EPSPs. $C 2$ shows synaptic responses to double stimulus of the OT at an interval of $4 \mathrm{msec}$. The onset of the EPSP induced by the second stimulus is indicated by a vertical broken line. Effects of 10 $\mu \mathrm{M}$ Bic and additional application of $50 \mu \mathrm{M}$ APV and $10 \mu \mathrm{M}$ CNQX are shown in D1-4 (faster time sweeps) and E1-4 (slower time sweeps). $F$ shows distribution of latencies of the EPSPs induced by the OT stimulation in SO neurons. Closed columns indicate the cases of wide-field vertical cells, and open columns indicate the other cell types.

was reduced by APV (Fig. 3D3,E3) and completely abolished after additional application of CNQX (Fig. 3D4,E4). This indicated that the EPSPs were mediated by both AMPA- and NMDA-type glutamate receptors. An interesting observation in these wide-field vertical cells in the SO was that when a depolarizing current pulse was applied to the soma via the recording pipette, the action potentials were generated at a threshold of $-58 \mathrm{mV}$ (Fig. $3 B$ ). In contrast, when the OT was stimulated, the action potential was induced, overriding the slow and small EPSP, at a threshold of approximately $-74 \mathrm{mV}$ when the resting membrane potential was $-76 \mathrm{mV}$ (Fig. 3 C1). Such spike generation insensitive to the somatic membrane potential was investigated in more detail. The records in Figure 4 show the synaptic responses of a wide-field vertical cell in the SO to the OT stimulation, when the membrane potential of the soma was set at different levels by varying the amplitude of the constantly injected current. Figure $4 A-D$ shows that the action potentials were generated from somatic membrane potentials of $-66,-76,-86$, and $-92 \mathrm{mV}$, when the membrane potentials were held at $-68,-78,-88$, and $-95 \mathrm{mV}$, respectively. Thus, the threshold for the spike generation was independent of the somatic membrane potential. As shown in Figure 4D, the M-spike (asterisk) was sometimes induced instead of the full-sized action potential. The amplitude of the EPSPs became slightly larger when the somatic membrane potential was held at an extremely hyperpolarized level $(-95 \mathrm{mV}$ in this case) than those induced from a more depolarized level $(-68 \mathrm{mV})$ (Fig. $4 D$, inset). Thus, the variation of the injected current somewhat affected the membrane potential at the synaptic sites where the EPSPs were induced and also at the sites where the action potentials were generated. However, this effect seemed minor. Figure $4 E$ shows that the action potential generated from the membrane potential below $-90 \mathrm{mV}$ was effectively blocked by application of $5 \mu \mathrm{M}$ CNQX and $50 \mu \mathrm{M}$ APV in all three neurons that were tested. Thus, the spike generation independent of the somatic membrane potential in the wide-field vertical cells was confirmed to be of synaptic origin but not of the antidromic origin. Such spike generation independent of the somatic membrane potential was observed in 13 of the 17 wide-field vertical cells in which monosynaptic EPSPs were observed after the OT stimulation. In the remaining four wide-field vertical cells and three SO neurons that were not identified as wide-field vertical cells, the action potentials were generated around the membrane potential of $-58 \mathrm{mV}$, overriding the peak of EPSPs as shown in the case of Figure $4 F 1$. These action potentials disappeared when the membrane potentials were hyperpolarized (to $-92 \mathrm{mV}$ in the cell shown in Fig. $4 F 2$ ) by increasing the amplitude of the constantly injected current.

A most probable explanation of such observation is that these 


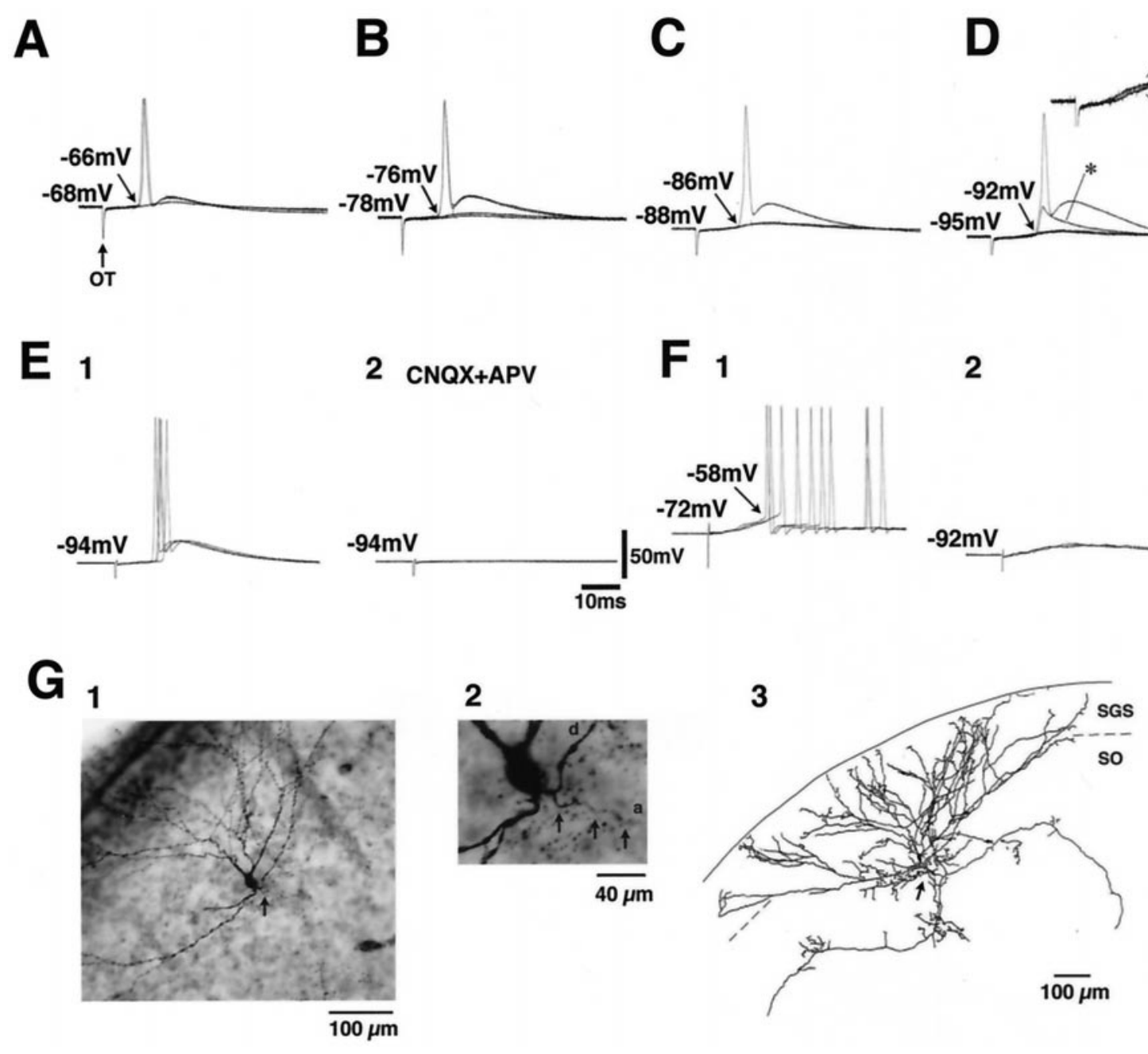

Figure 4. Synaptic responses and action potential generation in a wide-field vertical cell in the SO induced by the OT stimulation when the resting membrane potentials of the soma $\left(V_{\mathrm{m}}\right)$ were held at different levels: $V_{\mathrm{m}}=-68 \mathrm{mV}(A),-78 \mathrm{mV}(B),-88 \mathrm{mV}(C)$, and $-95 \mathrm{mV}(D)$, respectively. The asterisk in $D$ indicates an M-spike. The inset in $D$ indicates superimposition of EPSPs without the action potentials taken from records in $A$ and $D$. E1 and $E 2$ indicate the effect of $5 \mu \mathrm{M} C \mathrm{CQX}$ and $50 \mu \mathrm{M} \mathrm{APV}$ on the action potential generated from the somatic membrane potential below $-90 \mathrm{mV}$. F1 and $F 2$ indicate the example of records in wide-field vertical cells in which the action potentials were generated from normal threshold level $(-58 \mathrm{mV}$ in this case) overriding the EPSPs evoked by the OT stimulation ( $F 1)$, and action potentials disappeared when the membrane potential was hyperpolarized by increasing the intensity of constantly injected hyperpolarizing current $(F 2)$. $G$, Morphological characteristics of a wide-field vertical cell in the SO. Photomicrographs $(G 1, G 2)$ and camera lucida drawing $(G 3)$ of a wide-field vertical cell whose axon was originated from a proximal dendrite (arrows). $a$ and $d$ in $G 2$ indicate axon and dendrite, respectively.

wide-field vertical cells received the synaptic input from the OT on their distal dendrites, which led to induction of EPSPs with long rise time when recorded in the soma because of long electrotonic length (Rall, 1964). Because the extremely hyperpolarized somatic membrane potential did not affect the spike generation, we speculated that the EPSPs elicited in the dendrites might have induced nonlinear dendritic spikes, which directly activated the initial segment of the axon bypassing the soma, and generated the action potentials. This speculation is substantiated by the fact that in 7 of the 10 morphologically identified wide-field vertical cells, the axons were originated from a proximal portion of a dendrite, which is illustrated in photomicrographs and camera lucida drawings in Figure $4 G$ (arrows). In the remaining three cells, we could not determine whether the axons were originated from the soma or a dendrite. This morphological property of wide-field vertical cells has already been illustrated [Langer and Lund (1974), their Plate 8]. Furthermore, we investigated the morphology of 14 wide-field vertical cells, which we stained in our previous study without investigating the synaptic input (Saito and Isa, 1997), and could confirm that the axon was originated from a dendrite in eight cases. However, it should be noted that this is not the case for all the wide-field vertical cells, because in 3 of the 14 cases, we confirmed that the axons originated directly from the soma. In the remaining three cases, the location of the axon hillock could not be confirmed. According to these speculations, in the four wide-field vertical cells in which the action potential was generated at the normal threshold level (shown in Fig. 4F1), the EPSPs might have been induced in the dendrites that did not possess the axon or the axon was originated from the soma in those cells. However, the morphological evidence for these speculations has not been obtained yet.

\section{Input from the OT to neurons in the SGI}

The effect of the OT stimulation was investigated in 32 SGI neurons, and among them, 21 neurons were successfully stained with biocytin. They consisted of seven fusiform cells, four pyra- 


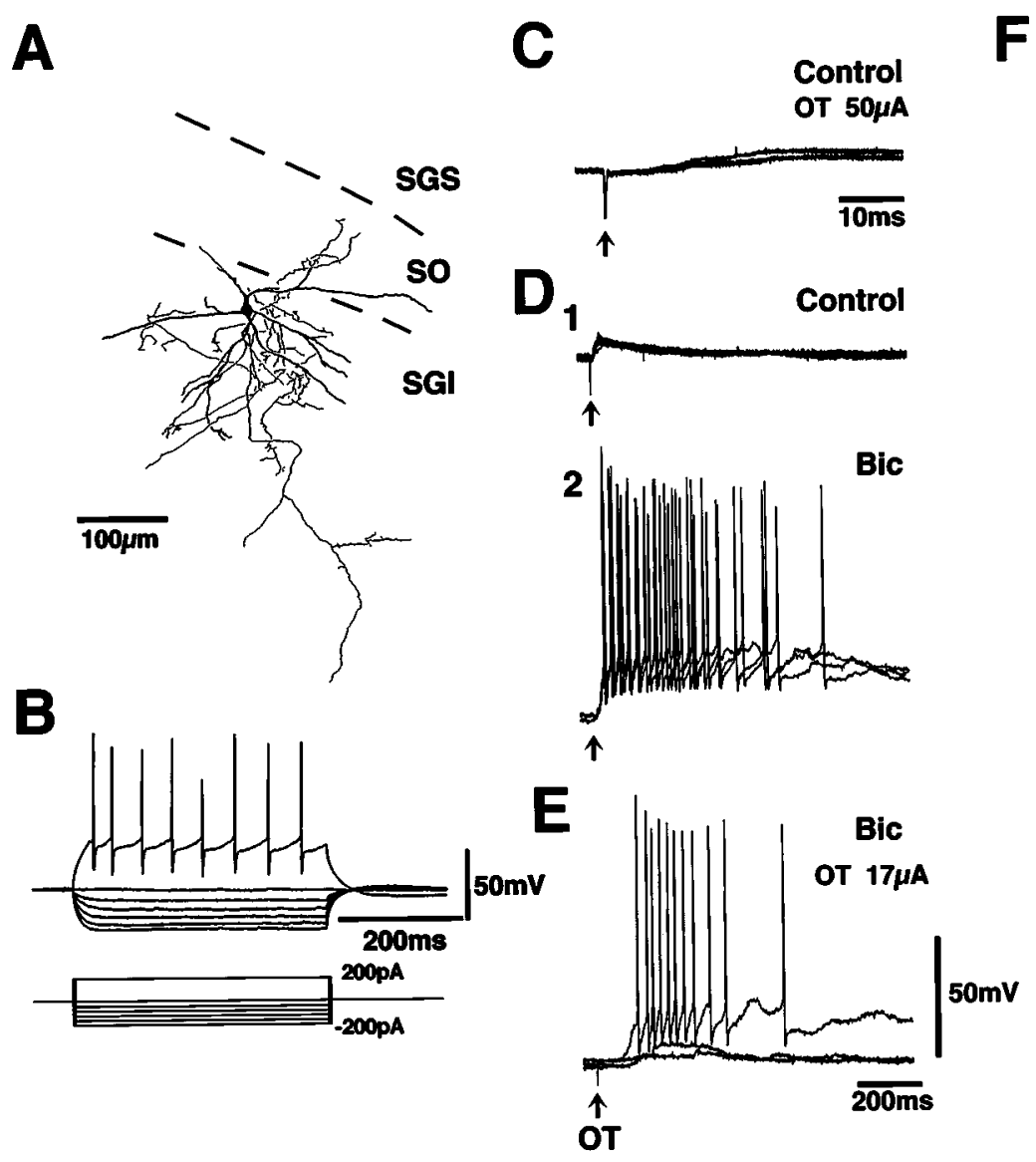

Figure 5. Synaptic potentials induced by the OT stimulation in an SGI neuron. The morphology of the recorded neuron, stained with biocytin, is illustrated in $A$. Dendrites are drawn as thick lines. The axon and its collaterals are drawn as thin lines. $B$ shows the voltage responses of this neuron to various current pulses. $C$ shows the synaptic responses of this neuron to the OT stimulation $(50 \mu \mathrm{A})$ in the control solution. $D$ shows effects of application of $10 \mu \mathrm{M} \mathrm{Bic} \mathrm{(D1,Control;} \mathrm{D2,} \mathrm{after} \mathrm{application} \mathrm{of} \mathrm{Bic).} \mathrm{E} \mathrm{shows} \mathrm{the} \mathrm{synaptic} \mathrm{responses} \mathrm{to} \mathrm{the} \mathrm{critical} \mathrm{stimulus} \mathrm{strength} \mathrm{(17} \mu$ A) for induction of long-lasting responses under the application of $10 \mu \mathrm{M}$ Bic. $F$ shows distribution of latencies of the EPSPs induced by the OT stimulation in SGI neurons. Open columns in $F$ indicate the EPSPs recorded in the slice preparation with a transection shown in Figure $6 \mathrm{~A}$.

midal cells, eight multipolar cells, one stellate cell, and one wide-field vertical cell according to the classification of Ma et al. (1990) and Langer and Lund (1974).

Figure 5 shows the results obtained from a multipolar cell in the SGI. In response to depolarizing current pulses, this neuron responded with repetitive firing with fairly constant intervals, whereas in response to hyperpolarizing current pulses, it showed inward rectification. This neuron had multipolar dendrites (thick lines) and dense axonal projections and terminations near the soma (thin lines). Stimulation of the OT induced EPSPs at latencies of 5.6-11.4 msec (Fig. 5C,D1). The onsets of the EPSPs fluctuated, suggesting that the EPSPs were induced by disynaptic or polysynaptic linkage. Application of Bic remarkably enhanced the late phase of the EPSPs, and the duration of EPSPs induced by a single stimulus often exceeded $500 \mathrm{msec}$ (Fig. 5D2). At the critical stimulus strength (17 $\mu \mathrm{A}$ in this case), the amplitude and duration of the EPSPs varied markedly from sweep to sweep, suggesting the existence of some nonlinear activating mechanism in the pathway to the SGI neurons (Fig. $5 E$ ). The EPSPs were observed in 24 of the 32 SGI neurons, and the latencies of the EPSPs ranged from 3.4 to $47.4 \mathrm{msec}$ (Fig. $5 F$, closed columns). In two neurons, a small EPSP component with short latency $(<5$ msec) occurred with fixed onset, and the possibility of monosynaptic EPSPs could not be excluded. However, they appeared to be minor among the present material. These results indicated the existence of disynaptic or polysynaptic excitatory pathways from the OT to SGI neurons and that the transmission in the pathway is under strong suppression by GABAergic neurons.

\section{The OT-SGS/SO-SGI pathway}

To further exclude the possibility of current spread to the structures outside the OT, a transection was made to the slice just medial to the stimulation site to spare the SGS and SO (Fig. 6A). Thirteen SGI neurons were recorded in seven slices with such manipulation. In this type of preparation, EPSPs could be induced in 11 SGI neurons by the OT stimulation at virtually the same latencies (Figs. 5F, open columns, 6C) at the submaximal stimulus strength for the conduction volley in the OT (Fig. 6B). The threshold for inducing EPSPs was $10-100 \mu \mathrm{A}$ in these neurons (below $50 \mu \mathrm{A}$ in nine neurons). In contrast to these preparations, the SGS and SO were selectively cut, and the OT was stimulated lateral to the transection in four slices (Fig. 6D). In these slices, seven SGI neurons were recorded. In the neuron shown in Figure $6 E, F$, the OT stimulation at $500 \mu \mathrm{A}$ did not induce the conduction volley of the OT recorded medial to the transection or the EPSPs in the SGI neurons. However, when the stimulus intensity was increased to $1 \mathrm{~mA}$, the conduction volley and the EPSPs started to appear simultaneously. The threshold 
A

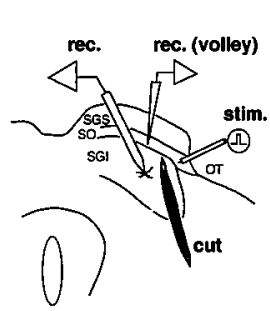

D

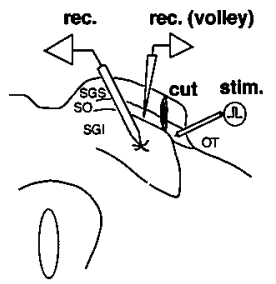

B

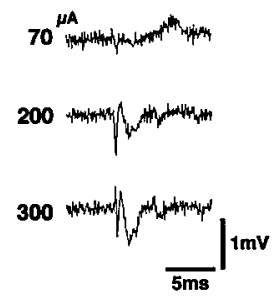

E

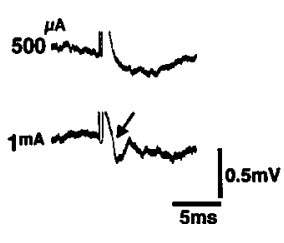

C Bic $10 \mu \mathrm{m}$
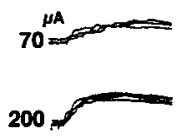
300

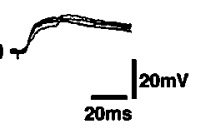

$F_{\text {Bic } 10 \mu \mathrm{M}}$

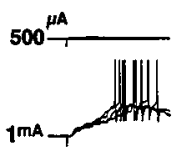

Figure 6. To exclude the possibility of current spread to the structures outside the OT, two types of transection were made to the slice as shown in $A$ and $D$. In $A$, a transection was made to spare the SGS and SO. $B$ shows the conduction volley in the OT recorded in the SO at various stimulation strengths in the preparation shown in $A$. Synaptic potentials under application of $10 \mu \mathrm{M}$ Bic at each stimulus strength are illustrated in $C$. In $D$, the SGS and SO were selectively transected, and the OT was stimulated lateral to the transection. Recordings of the conduction volley in the OT and synaptic potentials in the SGI neurons were made medial to the transection. $E$ and $F$ indicate the conduction volley in the OT and the EPSPs in the SGI neurons under application of $10 \mu \mathrm{M}$ Bic in the preparation shown in $D$, respectively. Note that an extremely strong stimulus $(1 \mathrm{~mA})$ was necessary to induce the conduction volley and the EPSPs.

for inducing EPSPs was $400 \mu \mathrm{A}-1 \mathrm{~mA}$ in four of the seven tested cells and above $1 \mathrm{~mA}$ in the remaining three cells after transection of the SGS and the SO. These results indicated that the current spread across the transection could occur when the stimulus intensity was increased above $400 \mu \mathrm{A}$, but this intensity was much higher than those that induced EPSPs in SGI neurons in slices with transections sparing the SGS and SO (described above). These results indicated that the EPSPs in the SGI neurons were induced by the stimulation of the OT, and the possibility of current spread to the structures outside the OT was excluded.

\section{Synaptic transmission from SGS to SGI neurons}

The effects of stimulation of the SGS were investigated in seven SGI neurons located ventral to the stimulation site (Fig. 7A). Stimulation of the SGS induced EPSPs in the SGI neurons (Fig. $7 B$ ) in the standard Ringer's solution in five of seven cells tested. The thresholds for inducing EPSPs were often below $10 \mu \mathrm{A}$. The latencies of the EPSPs ranged from 3.8 to $20.0 \mathrm{msec}(n=5)$. When the latency was short, the onsets of the EPSPs were constant from sweep to sweep (Fig. 7B3). Thus, the minimal synaptic linkage was estimated as monosynaptic. These EPSPs were much enhanced by application of Bic (Fig. 7C2). The late component of the EPSPs was largely abolished by application of APV (Fig. $7 C 3$ ), and the remaining fast component was abolished by additional application of CNQX (Fig. 7C4). In the remaining two SGI neurons, stimulation of the SGS did not induce EPSPs in the standard Ringer's solution, but long-latency EPSPs (14.3 msec in this case) could be observed after application of Bic, suggesting lack of monosynaptic linkage but existence of a polysynaptic

pathway from the SGS to these SGI neurons (Fig. 7D1,D2). In these cases, additional application of APV completely abolished the polysynaptic EPSPs (Fig. 7D3), suggesting that the activation of NMDA receptors was essential for bringing the membrane potential of intercalated neurons above the firing threshold level. All these results have suggested that excitatory synaptic transmission from the SGS to SGI neurons is mediated by both AMPAand NMDA-type glutamate receptors, and among them, NMDA receptors make a significant contribution.

\section{DISCUSSION}

This study presented electrophysiological evidence confirming the existence of the excitatory pathway from the OT to SGI neurons via neurons in the SGS and SO in rats. The excitatory synaptic transmission in this pathway is mediated by both AMPAand NMDA-type glutamate receptors and is under strong suppression by the GABAergic system. We believe that this basic circuit demonstrated in the present study using young rats (P1724) will remain in adult animals; however, we have to keep in mind that the synaptogenesis in the SC reaches its peak at P14 and continues until P30 (Warton and McCart, 1989), and accordingly development of the SC is not completed in the animals we used.

\section{Optic fiber input to the SGS neurons}

Visual synaptic transmission in the SGS has been studied by Binns and Salt (1994) in anesthetized cats by a combination of extracellular unit recording and iontophoretic application of neurotransmitter antagonists. These authors observed that both NMDA- and non-NMDA-type glutamate receptors contribute to visual synaptic transmission in the SGS. In the present study, we used the SC slices obtained from rats, and the appropriate stimulus strength for selective activation of the OT was determined by measuring the stimulus strength that induces the maximal conduction volley in the OT. This experimental preparation enabled detailed analysis of the synaptic organization of the OT-SGS synapses using the whole-cell patch-clamp technique. Furthermore, combining intracellular staining with biocytin enabled us to identify recorded cells. Previous morphological studies have revealed that SGS neurons are composed of several subclasses (Sterling, 1971; Langer and Lund, 1974; Norita, 1980). In the present study, we recorded from at least five subclasses of neurons, and those that received monosynaptic excitation included piriform cells, stellate cells, and narrow-field vertical and horizontal cells.

\section{Visual synaptic input to wide-field vertical cells in the SO}

The present results showed that wide-field vertical cells in the SO receive monosynaptic excitatory input from the optic tract on their dendrites. We found that the spike generation of the widefield vertical cells is insensitive to the somatic membrane potential and considered two possible explanations. (1) As described in Results, the EPSP induced in the distal dendrites evoked a dendritic spike that directly triggered the spike generation at the initial segment of the axon originated from one of the dendrites, bypassing the soma. (2) The OT axons made synapses on the axon of the wide-field vertical cell. Morphological examinations are necessary to obtain a final conclusion. Furthermore, generation of dendritic spikes or synaptic contact on the axon should be examined by direct recording from dendrites or axons or both. If possibility (1) is correct, it suggests that all the dendrites extended from the wide-field vertical cells are not equivalent, but rather the one that carries the axon has a dominant effect on generation of 
A

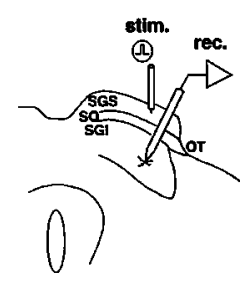

$B_{1}$

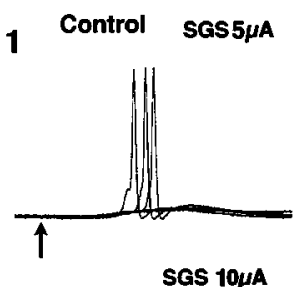

2

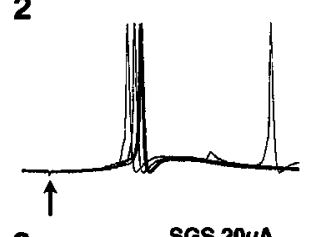

3

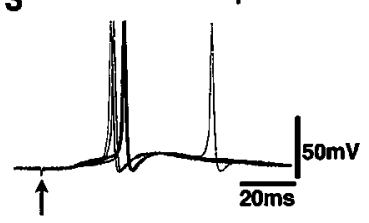

C

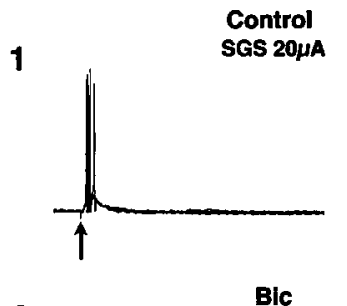

2

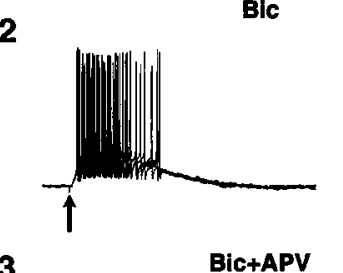

3
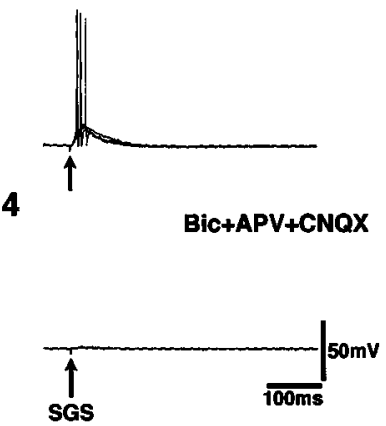

D

1

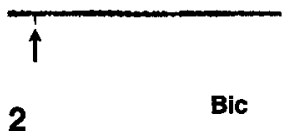

2
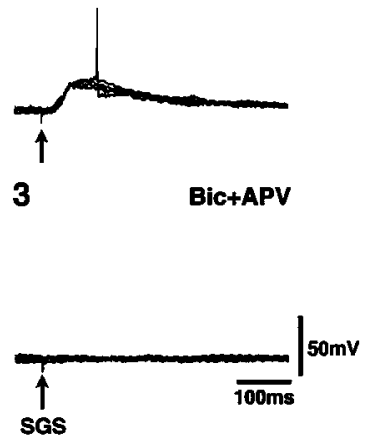

Figure 7. Effect of stimulation of the SGS on an SGI neuron. Experimental arrangement is shown in $A$. $B$ shows the EPSPs induced by the OT stimulation at different stimulus strengths in the control solution. The effect of 10 $\mu \mathrm{M}$ Bic on the EPSP (compare $C 1$ and $C 2$ ) and of additional application of 50 $\mu \mathrm{M}$ APV (C3) and of $50 \mu \mathrm{M}$ APV plus $10 \mu \mathrm{M}$ CNQX (C4). $D$ shows records from another SGI neuron. The OT stimulation did not induce EPSPs in the control solution (D1); however, it induced long-latency EPSPs under application of $10 \mu \mathrm{M}$ Bic (D2), which was completely abolished by application of $50 \mu \mathrm{M}$ APV (D3). action potential over the others. Such a phenomenon has also been observed in dopaminergic neurons in the substantia nigra (Hausser et al., 1995). Thus, extensive projection of dendrites from the wide-field vertical cells may suggest that these neurons possess a wide visual receptive field; however, inputs on individual dendrites are not equivalent, and the magnitude of visual response of these neurons may vary depending on which dendrite received the input.

\section{Connection from the OT to the SGI}

The present study showed that stimulation of the OT induced disynaptic or polysynaptic EPSPs in SGI neurons. Thus, the excitatory pathway from the OT to SGI neurons exists in the local circuit of the SC. Morphological analysis and electrophysiological recordings from SGS/SO neurons revealed possible neuronal elements comprising the pathway. Intracellular staining with biocytin revealed that most SGS neurons projected abundant axon collaterals and terminal bouton-like structures in the ventral part of the SGS and the SO, but the number of bouton-like structures decreased in the SGI. On the other hand, the wide-field vertical cells in the SO extended divergent dendritic trees into the SGS and projected axons and terminal bouton-like structures in the ventral part of the SO and in the SGI. Therefore, wide-field vertical cells in the SO can mediate indirect (via SGS neurons) visual input to neurons in the SGI neurons. These neurons can also mediate direct visual input to the SGI neurons, because they receive monosynaptic excitatory input from the OT on their dendrites. Furthermore, some of the SGI neurons extended dendrites into the SO and sometimes farther dorsally to the SGS (Lopez-Barneo and Llinás, 1988; Mooney et al., 1988; Hall and Lee, 1993; Saito and Isa, 1997). This morphological evidence has suggested that SGI neurons receive indirect visual input from SGS neurons mainly on their dendrites. Although some SGI neurons could receive direct input from the OT on their dendrites in the SGS/SO (Mooney et al., 1988), the direct connection from the OT to SGI neurons appeared to be minor because monosynaptic EPSPs were observed only in a small population of SGI neurons in the present study.

It is not clear which type of SGS neurons is excitatory or inhibitory. We have shown that various types of SGS neurons receive monosynaptic excitatory input from the OT. Although Mize (1992) showed that GABAergic neurons in the SGS include horizontal, stellate, and piriform cells, it is not yet clear whether all neurons of these subclasses are inhibitory. Thus, until it is revealed which subclass of the SGS neurons with excitation from the OT is excitatory, it will not be clear which subclass of SGS neuron mediates the excitatory synaptic transmission from the OT to the SO and SGI neurons.

\section{Regulation of signal transmission from the SGS/SO to the SGI}

Freeman and Singer (1983) analyzed the synaptic input from the OT to the SC by current source density analysis in anesthetized cats. These authors showed that the synaptic current was induced mainly in the SGS, but no large synaptic currents appeared to be induced in the SGI. This suggested that the neural connection from the SGS or SO to the SGI is not strong enough to elicit large synaptic currents in SGI neurons in intact cats. However, the present study clarified that the synaptic transmission from the OT to the SGI neurons is under strong regulation by GABAergic system, because application of bicuculline predominantly enhanced the EPSPs recorded in the SGI neurons (Fig. 5D). The importance of GABAergic input in controlling the output from the SC has been shown by Hikosaka and Wurtz (1985), who reported that injection of bicuculline remarkably increased the occurrence of express saccades. 
Thus, a possible explanation of the mechanisms to generate express saccades is that the signal transmission from the SGS/SO to the SGI neurons is suppressed in cases of regular saccades; however, the transmission becomes facilitated, for example, in cases of express saccades, possibly by disinhibition from GABAergic neurons.

\section{Nonlinear activation mechanisms of SGI neurons}

A unique property of the neural circuit in the SC is that the output from the circuit has a nonlinear relationship with its input. Induction of saccadic eye movements has an all-or-none relationship with the stimulus strength of the SC (Robinson, 1972; Schiller and Stryker, 1972). The present study showed that the EPSPs induced by the OT stimulation were remarkably enhanced by application of bicuculline. The enhanced EPSPs often lasted $>500 \mathrm{msec}$. Such a phenomenon was not observed in SGS/SO neurons and appeared to be a unique property of the SGI neurons. These results suggested the existence of some nonlinear regenerative property in the pathway to the SGI. The present study showed that NMDA-type glutamate receptors play an important role in mediating the signal transmission to the SGI neurons (Fig. 7E). NMDA-type glutamate receptors have negative slope conductance below $-30 \mathrm{mV}$ of membrane potential. In this voltage range, the more the cell is depolarized, the larger the current that passes through the NMDA-receptor channels; thus the regenerative process can be switched on. In addition, intrinsic properties of individual neurons comprising the local circuit may contribute to the nonlinear property of the circuit (Saito and Isa, 1997). The actual contribution of these elements should be examined experimentally either in vivo or in vitro.

\section{REFERENCES}

Behan M, Appel PP (1992) Intrinsic circuitry in the cat superior colliculus: projection from the superficial layers. J Comp Neurol 315:230-243.

Binns KE, Salt TE (1994) Excitatory amino acid receptors participate in synaptic transmission of visual responses in the superficial layers of the cat superior colliculus. Eur J Neurosci 6:161-169.

Cynader M, Berman N (1972) Receptive-field organization of monkey superior colliculus. J Neurophysiol 35:187-201.

Edwards FA, Konnerth A, Sakmann B, Takahashi T (1989) A thin slice preparation for patch clamp recordings from neurones of the mammalian central nervous system. Pflügers Arch 414:600-612.

Edwards SB (1980) The deep cell layers of the superior colliculus: their reticular characteristics and structural organization. In: The reticular formation revisited (Hobson JA, Brazier MAB, eds), pp 193-209. New York: Raven.

Fischer B (1987) The preparation of visually guided saccades. Rev Physiol Biochem Pharmacol 106:1-35.

Fischer B, Boch R (1983) Saccadic eye movements after extremely short reaction times in the monkey. Brain Res 260:21-26.

Fischer B, Ramsperger E (1984) Human express saccades: extremely short reaction times of goal directed eye movements. Exp Brain Res 57:191-195.

Freeman B, Singer W (1983) Direct and indirect visual inputs to superficial layers of cat superior colliculus: a current source-density analysis of electrically evoked potentials. J Neurophysiol 49:1075-1091.

Hall WC, Lee P (1993) Interlaminar connections of the superior colliculus in the tree shrew. I. The superficial gray layer. J Comp Neurol 332:213-223.

Hamill OP, Marty A, Neher E, Sakmann B, Sigworth FJ (1981) Improved patch clamp techniques for high-resolution current recording from cells and cell free patches. Pflügers Arch 391:85-100.

Harting JK, Updyke BV, Van Lieshout DP (1992) Corticotectal projec- tions in the cat: anterograde transport studies of twenty-five cortical areas. J Comp Neurol 324:379-414.

Harvey AR, Worthington DR (1990) The projection from different visual cortical areas to the rat superior colliculus. J Comp Neurol 298:281-292.

Hausser M, Stuart G, Racca C, Sakmann B (1995) Axonal initiation and active dendritic propagation of action potentials in substantia nigra neurons. Neuron 15:637-647.

Hikosaka O, Wurtz RH (1985) Modification of saccadic eye movements by GABA-related substances. I. Effect of muscimol and bicuculline in monkey superior colliculus. J Neurophysiol 53:266-291.

Horikawa K, Armstrong WE (1988) A versatile means of intracellular labeling: injection of biocytin and its detection with avidine conjugates. J Neurosci Methods 25:1-11.

Huerta MF, Harting JK (1982) Tectal control of spinal cord activity: neuroanatomical demonstration of pathways connecting the superior colliculus with the cervical spinal cord grey. Prog Brain Res 57:293-328.

Langer TP, Lund RD (1974) The upper layers of the superior colliculus of the rat: a Golgi study. J Comp Neurol 158:405-436.

Lee P, Hall WC (1995) Interlaminar connections of the superior colliculus in the tree shrew. II: Projections from the superficial gray to the optic layer. Vis Neurosci 12:573-588.

Lee PH, Helms MC, Augustine GJ, Hall WC (1997) Role of intrinsic synaptic circuitry in collicular sensorimotor integration. Proc Natl Acad Sci USA 94:13299-13304.

Lopez-Barneo J, Llinás R (1988) Electrophysiology of mammalian tectal neurons in vitro. I. Transient ionic conductances. J Neurophysiol 60:853-868.

Ma TP, Cheng H-W, Czech JA, Rafols JA (1990) Intermediate and deep layers of the macaque superior colliculus: a Golgi study. J Comp Neurol 295:92-110.

Mize RR (1992) The organization of GABAergic neurons in the mammalian superior colliculus. Prog Brain Res 90:219-248.

Mooney RD, Nikoletseas MM, Hess PR, Allen Z, Lewin AC, Rhoades RW (1988) The projection from the superficial layer to the deep layers of the superior colliculus: an intracellular horseradish peroxidase injection study in the hamster. J Neurosci 8:1384-1399.

Mooney RD, Huang X, Rhoades RW (1992) Functional influence of interlaminar connections in the hamster's superior colliculus. J Neurosci $12: 2417-2432$.

Norita M (1980) Neurons and synaptic patterns in the deep layers of the superior colliculus of the cat. A Golgi and electron microscopic study. J Comp Neurol 190:29-48.

Rall W (1964) Time constants and electrotonic length of membrane cylinders and neurons. Biophys J 9:1483-1508.

Rhoades RW, Mooney RD, Rohrer WH, Nikoletseas MM, Fish SE (1989) Organization of the projection from the superficial to the deep layers of the hamster's superior colliculus as demonstrated by the anterograde transport of Phaseolus vulgaris leucoagglutinin. J Comp Neurol 283:54-70.

Robinson DA (1972) Eye movements evoked by collicular stimulation in the alert monkey. Vision Res 12:1795-1808.

Saito Y, Isa T (1997) Local circuit of the superior colliculus in rats. I. Intrinsic firing and morphological properties of neurons in the optic and intermediate layers. Soc Neurosci Abstr 510.14:1297.

Schiller PH, Stryker M (1972) Single-unit recording and stimulation in superior colliculus of the alert rhesus monkey. J Neurophysiol 35:915-924.

Sprague JM (1975) Mammalian tectum: intrinsic organization, afferent inputs, and integrative mechanisms. In: Sensorimotor function of the midbrain tectum, Neurosciences Research Program Bulletin, Vol 13 (Ingle D, Sprague JM, eds), pp 204-214. Cambridge, MA: MIT.

Sterling P (1971) Receptive fields and synaptic organization of the superficial gray layer of the cat superior colliculus. Vision Res [Suppl] 3:309-328.

Wallace MT, Stein BE (1996) Sensory organization of the superior colliculus in cat and monkey. Prog Brain Res 112:301-311.

Warton SS, McCart R (1989) Synaptogenesis in the stratum griseum superficiale of the rat superior colliculus. Synapse 3:136-148. 